\title{
Enhanced visible-light-driven photocatalytic performance of porous graphitic carbon nitride
}

\author{
Fei Chang ${ }^{\mathrm{a}, *}$, Chenlu $\mathrm{Li}^{\mathrm{a}}$, Jieru Luo ${ }^{\mathrm{a}}$, Yunchao Xie ${ }^{\mathrm{a}}$, Baoqing Deng ${ }^{\mathrm{a}}$, Xuefeng $\mathrm{Hu}^{\mathrm{b}, *}$ \\ a School of Environment and Architecture, University of Shanghai for Science and Technology, Shanghai 200093, PR China \\ ${ }^{\mathrm{b}}$ Key Laboratory of Coastal Environmental Processes and Ecological Remediation, Yantai Institute of Coastal Zone Research, Chinese Academy of Sciences, \\ Yantai, Shandong 264003, PR China
}

\section{A R T I C L E I N F O}

\section{Article history:}

Received 28 May 2015

Received in revised form 13 August 2015

Accepted 15 August 2015

Available online 19 August 2015

\section{Keywords:}

Porous

$g-\mathrm{C}_{3} \mathrm{~N}_{4}$

Nitric acid

Photocatalysis

Rhodamine B

Mechanism

\begin{abstract}
A B S T R A C T
In this study, a series of porous graphitic carbon nitride $\left(g-\mathrm{C}_{3} \mathrm{~N}_{4}\right)$ materials were fabricated through a direct pyrolysis of protonated melamine by nitric acid solution. These as-prepared porous samples were characterized by a collection of analytical techniques. It was found that a proper concentration of nitric acid solution involved facilitated to generate samples in tube-like morphology with numerous pores, identified with X-ray diffraction patterns, FT-IR spectra, SEM, TEM, and BET measurements. These $g$ $\mathrm{C}_{3} \mathrm{~N}_{4}$ samples were subjected to photocatalytic degradation of dye Rhodamine $\mathrm{B}(\mathrm{RhB})$ in aqueous under visible-light irradiation. Under identical conditions, those porous $g-C_{3} N_{4}$ samples showed significantly improved catalytic performance in comparison with the sample prepared without the introduction of nitric acid. In particularly, the best candidate, sample M1:1, showed an apparent reaction rate nearly 6.2 times that of the unmodified counterpart. The enhancement of photocatalytic performance could be attributed to the favorable porous structure with the enlarged specific surface area and the suitable electronic structure as well. In addition, ESR measurements were conducted for the sake of proposing a photocatalytic degradation mechanism.
\end{abstract}

(c) 2015 Elsevier B.V. All rights reserved.

\section{Introduction}

Polymeric $\mathrm{g}-\mathrm{C}_{3} \mathrm{~N}_{4}$ materials, regarded as a family of attractive non-metal semiconductors, possess two-dimensional $\pi$ conjugated structures that are composed of numerous tri-s-triazine units [1]. These units mainly consist of $\mathrm{sp}^{2}$-hybridized carbon and nitrogen atoms connected by covalence bonds, ensuring unique chemical and thermal stabilities. With favorable structural and electronic properties, $g-\mathrm{C}_{3} \mathrm{~N}_{4}$ samples are able to catalyze the splitting $\mathrm{H}_{2} \mathrm{O}$ to generate gaseous oxygen and hydrogen, and decompose various organic contaminants under visible-light illumination, hereby attracting intensive attention in fields of environmental remediation and energy storage [2-4].

However, the photocatalytic efficiency of $g-C_{3} \mathrm{~N}_{4}$ semiconductors is relatively low and still far from the level required for real applications, mainly owing to the small surface area values and high recombination rate of photogenerated electron-hole pairs [5-8]. As a result, many efforts have been devoted to modify synthetic procedures toward $g-C_{3} N_{4}$ to gain an efficient photocatalytic

\footnotetext{
* Corresponding authors.

E-mail addresses: feichang@usst.edu.cn (F. Chang),xfhu@yic.ac.cn (X. Hu).
}

system, such as doping with metal oxides or nonmetal elements [9-13], construction of porous or hierarchical nanostructures [14-16], and combining other semiconductors with suitable band structures [17-22]. Among strategies mentioned above, the fabrication of porous $g-\mathrm{C}_{3} \mathrm{~N}_{4}$ from the precursor of prononated melamine is quite promising and possible to achieve satisfactory catalytic efficiency. Dong demonstrated the porous $g-\mathrm{C}_{3} \mathrm{~N}_{4}$ produced from $\mathrm{HCl}$-treated melamine possessed the extraordinarily large specific surface area and displayed excellent photocatalytic ability [23]. Yan reported the enhanced photocatalytic performance of porous $g$ $\mathrm{C}_{3} \mathrm{~N}_{4}$ prepared by the pyrolysis of protonated melamine by $\mathrm{H}_{2} \mathrm{SO}_{4}$ solution [24]. Gao found that the nanotubular $g-\mathrm{C}_{3} \mathrm{~N}_{4}$ by pyrolysis of $\mathrm{HNO}_{3}$-treated melamine displayed intense photoluminescence emission and reproducible photoconductivity [25]. Using a similar procedure, Tahir prepared porous $g-\mathrm{C}_{3} \mathrm{~N}_{4}$ samples with the improved catalytic behavior under visible-light illumination [26]. Therefore, it is realized that the sintering of melamine pretreated in an acidic environment, especially in a nitric acid solution, tends to supply porous $g-C_{3} N_{4}$ materials with favorable physicochemical properties, thus guaranteeing excellent photocatalytic capability [26-28].

In this investigation, we dispersed the melamine powder in aqueous solution, instead of the generally used solvent ethylene 
glycol that was suspected to be of low toxicity [29]. After treated with nitric acid aqueous solution, the melamine was directly heated to produce porous $g-\mathrm{C}_{3} \mathrm{~N}_{4}$ samples that were analyzed by various physicochemical techniques. The concentration of nitric acid added exerted an important role on the structure of obtained $g$ $\mathrm{C}_{3} \mathrm{~N}_{4}$ samples. In the presence of an appropriate concentration of nitric acid, tube-like porous $g-C_{3} \mathrm{~N}_{4}$ samples with enlarged specific surface areas were achieved. These samples were then subjected to photocatalytic evaluation toward decomposition of RhB under visible light irradiation. As expected, modified samples showed the enhanced photocatalytic capabilities. In particularly, the best candidate, sample $M 1: 1$, could show the apparent reaction rate nearly 6.2 times that of unmodified $g-C_{3} \mathrm{~N}_{4}$. The enhancement of photocatalytic efficiency could be ascribed to a synergistic effect of the porous microstructures with enlarged specific surface areas and suitable band gap energies. In addition, a possible mechanism was finally proposed basing upon ESR measurements.

\section{Materials and methods}

\subsection{Reagents}

Melamine $\left(\mathrm{C}_{3} \mathrm{H}_{6} \mathrm{~N}_{3}, \mathrm{CP}\right)$, isopropanol (IPA, AR), disodium ethylenediaminetetraacetate dehydrate (EDTA-2Na, AR), terephthalic acid (TA, AR), 1,4-benzoquinone (BQ, AR), Rhodamine B (RhB, $A R$ ), and other chemicals involved were purchased from Sinopharm Chemical Reagent Co., Ltd. (China) and used directly for experimental without further purifications. All aqueous solutions throughout were prepared with the deionized water.

\subsection{Preparation of porous $\mathrm{g}-\mathrm{C}_{3} \mathrm{~N}_{4}$ samples}

In a typical procedure, a fixed amount of melamine was dispersed into deionized water $(50 \mathrm{~mL})$ and stirred for $1 \mathrm{~h}$. A desired amount of concentrated nitric acid was dissolved into deionized water $(30 \mathrm{~mL})$ to make an aqueous solution that was then dropped slowly into the above the melamine aqueous suspension. The powder obtained by the filtration, rinse with water and absolute ethanol, and dryness at $60^{\circ} \mathrm{C}$ was transferred into a covered crucible and stood at $500^{\circ} \mathrm{C}$ for $4 \mathrm{~h}$, with a heating rate of $10^{\circ} \mathrm{C} / \mathrm{min}$. After cooling down, these samples were collected and nominated as M6:1, M3:1, and M1:1, accordingly to molar ratios of melamine $/ \mathrm{HNO}_{3}$. $g-\mathrm{C}_{3} \mathrm{~N}_{4}$ was prepared using a same procedure without addition of $\mathrm{HNO}_{3}$. Another sample was also synthesized. When the nitric acid solution was dropped into the melamine aqueous solution, a white precipitate was generated in a quite quick manner, resulting into a shaking white block in which the magnetic was covered. The white block was underwent a same procedure described above to supply a sample, named M1:3.

\subsection{Characterization}

X-ray diffraction patterns (XRD) were recorded on a Bruker D8 Advance $\mathrm{X}$-ray diffractometer using a $\mathrm{Cu} \mathrm{K} \alpha$ radiation source $(\lambda=1.5406 \AA)$. The general morphology of samples was measured on a scanning electron microscopy (SEM, XL-30, Philips) and transmission electron microscopy (TEM, JEOLJEM-2010, Japan) operated at $200 \mathrm{kV}$. The FT-IR spectra were conducted on a Bruker V-70 Fourier transformed infrared spectrophotometer using a $\mathrm{KBr}$ pellet as the reference. X-ray photoelectron spectroscopy (XPS) and the valence-band X-ray photoelectron spectroscopy (VB XPS) measurements were performed by using a Thermo ESCALAB 250Xi System. Binding energies were calibrated by using the containment carbon $(\mathrm{C} 1 \mathrm{~s}=284.8 \mathrm{~V})$. The specific surface areas were measured using the BET method by nitrogen adsorption-desorption isotherms at $77 \mathrm{~K}$ using an automatic surface are and size analyzer (3H-2000PS2). All samples were degassed at $473 \mathrm{~K}$ for $2 \mathrm{~h}$ prior to analysis. Elemental analysis (EA) was carried out on an Elemental Vario III elemental analyzer. UV-vis diffuse reflectance spectra (UV-vis DRS) were obtained on a Hitachi U-4100 spectrophotometer using $\mathrm{BaSO}_{4}$ as a reference.

\subsection{Photocatalytic measurements}

Photocatalytic capabilities of obtained samples were evaluated toward degradation of dye $\mathrm{RhB}$ under irradiation from a $300 \mathrm{~W}$ Xe lamp (CEL-HXF300, AuLight, Beijing) equipping with a $420 \mathrm{~nm}-780 \mathrm{~nm}$ cut-off filter at the light source. Typically, the photocatalyst $(40 \mathrm{mg}$ ) was dispersed in an aqueous solution of $\mathrm{RhB}\left(8 \mathrm{mg} \mathrm{L}^{-1}, 80 \mathrm{~mL}\right)$ with a magnetic stirrer and the distance between the light source and the surface of reaction suspension was fixed at $20 \mathrm{~cm}$. The suspension was magnetically stirred for $0.5 \mathrm{~h}$ in dark to ensure an adsorption-desorption equilibrium before exposing to the visible-light irradiation. At every $20 \mathrm{~min}$ intervals, $5 \mathrm{~mL}$ aliquot was sampled and centrifuged to remove suspended catalyst particles. The concentration of RhB was analyzed by a UV-vis spectrophotometer (Purkinje General T6) at $554 \mathrm{~nm}$.

ESR signals of active radicals spin-trapped by 5,5-dimethyl1-pyrroline-N-oxide (DMPO) were examined with a JES-FA200 spectrometer. The settings for the spectrometer were: center field, $323.357 \mathrm{mT}$; microwave frequency, $9055.619 \mathrm{MHz}$; power, $0.998 \mathrm{~mW}$.

\section{Results and discussion}

The crystallographic phase and compositions of as-synthesized g- $\mathrm{C}_{3} \mathrm{~N}_{4}$ samples were analyzed by XRD, as depicted in Fig. 1a. It is obvious that melamine could be converted to $g-C_{3} N_{4}$ phase by means of pyrolysis whether the nitric acid was involved or not, which is proven by the presence of both characteristic diffraction peaks of pure $g-C_{3} N_{4}$. The intense peak centered at $27.7^{\circ}$ is assigned to the crystallographic plane (002), an interlayer stacking of the conjugated aromatic segments. The calculated interlayer distance of aromatic units $(0.322 \mathrm{~nm})$ is relatively smaller than those of crystalline $g-\mathrm{C}_{3} \mathrm{~N}_{4}$ reported [3]. A weak peak appears at around $13.2^{\circ}$ and is attributed to the plane $(100)$, relating to the in-plane structural repeating motif. Further observation of Fig. 1a indicates that the intensity of this reflection peak becomes gradually reduced as the concentration of $\mathrm{HNO}_{3}$ solution increases, possibly owing to the reduced planar size of layers induced by the calcination process when $\mathrm{HNO}_{3}$ molecules was present [8]. Gao reported that a white precipitate was formed as soon as the $\mathrm{HNO}_{3}$ aqueous was charged into the ethylene glycol solution containing melamine. Components in the precipitate were determined as protonated melamine and $\mathrm{NO}_{3}{ }^{-}$species by means of elemental analyses [25]. In our case, we attempted a similar synthetic procedure except that the solvent ethylene glycol was replaced by deionized water, in the interest of avoiding the usage of low-toxic solvent. During reaction, a white precipitate was formed and increased with the increase of the nitric acid concentration. It is reasonable to deduce that the components in the white precipitate are the same as those in Gao's report. During sintering, the $\mathrm{HNO}_{3}$ contained in precipitate activated the melamine to convert to $g-\mathrm{C}_{3} \mathrm{~N}_{4}$ phase via releasing massive gaseous $\mathrm{NH}_{3}$ and $\mathrm{CO}_{2}$. In addition, we speculate the $\mathrm{HNO}_{3}$ molecules were unstable at high temperature and readily decomposed to various gases, like molecular oxygen and nitrogen oxides. These gases together with $\mathrm{NH}_{3}$ and $\mathrm{CO}_{2}$ could create an oxidative environment and partially destroy the microstructure of $g-\mathrm{C}_{3} \mathrm{~N}_{4}$ to generate some randomly aggregated fragments or nanosheets, resulting into the destruction of in-plane structural repeating motif 

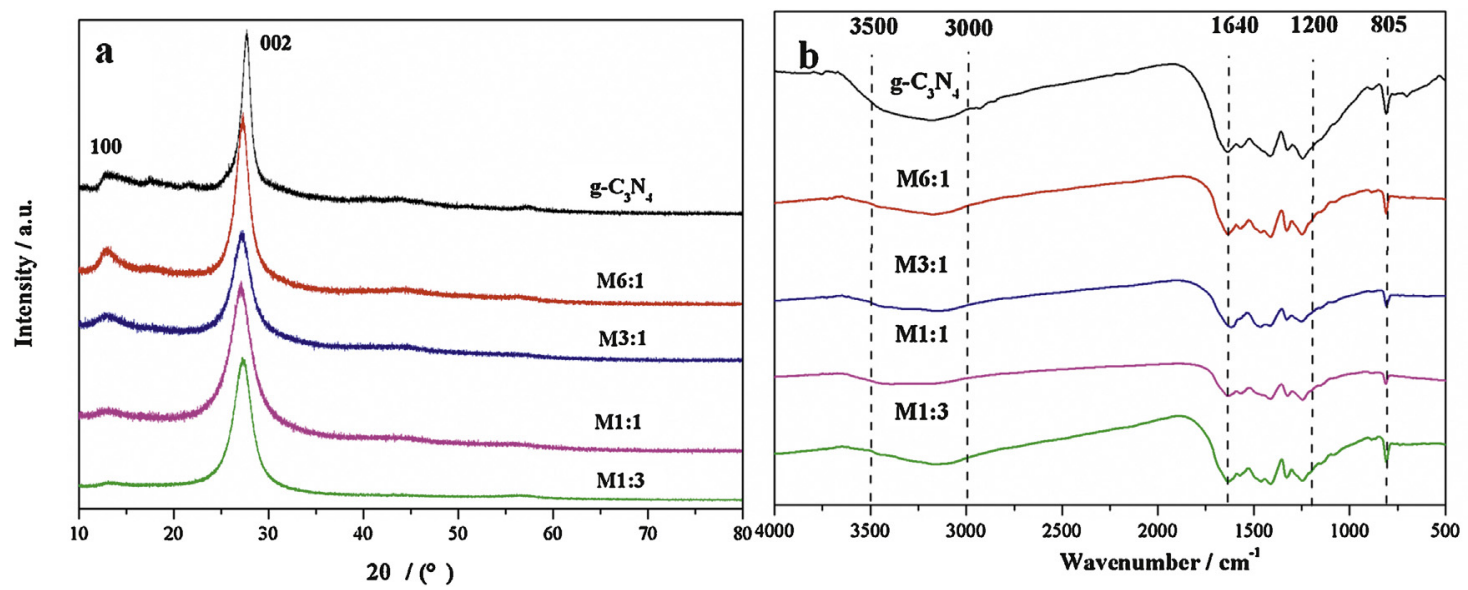

Fig. 1. XRD patterns (a) and FT-IR spectra (b) of as-synthesized $g-C_{3} N_{4}$ samples.

and thus the low intensity of diffraction peak at $13.2^{\circ}$ in XRD patterns, similar to the phenomenon in our previous work when the mixture of $\mathrm{NaNO}_{3}$ and melamine was annealed [30]. The exact role of $\mathrm{HNO}_{3}$ in the calcination process is not clear and need for further study.

FT-IR spectra of obtained $g-\mathrm{C}_{3} \mathrm{~N}_{4}$ samples are shown in Fig. $1 \mathrm{~b}$. The intense adsorption band ranging from $1200 \mathrm{~cm}^{-1}$ to $1640 \mathrm{~cm}^{-1}$ is relevant to the typical skeletal stretching vibration of $\mathrm{CN}$ heterocycles. The sharp peak at $805 \mathrm{~cm}^{-1}$ is linked to the characteristic breathing mode of triazine units [31]. The wide adsorption band between $3000 \mathrm{~cm}^{-1}$ and $3500 \mathrm{~cm}^{-1}$ is indexed to the stretching vibration of residual $\mathrm{N}-\mathrm{H}$ or the $\mathrm{O}-\mathrm{H}$ bonds, originating from uncondensed amino groups or adsorbed water on surface [32]. The presence of above characteristic peaks verifies the formation of $g-\mathrm{C}_{3} \mathrm{~N}_{4}$ phase even the precursor melamine was treated with $\mathrm{HNO}_{3}$ aqueous solution [33].

The surface chemical composition and valence band states of sample M1:1 were analyzed by XPS spectra, as shown in Fig. 2. The $\mathrm{C} 1 \mathrm{~s}$ signal was calibrated at $284.8 \mathrm{eV}$. The survey spectrum reveals the existence of elements $\mathrm{C}, \mathrm{N}$, and $\mathrm{O}$. The $\mathrm{C} 1 \mathrm{~s}$ peaks can be fitted with three peaks at binding energies of $284.8,286.2$, and $288.2 \mathrm{eV}$, indicative of three different chemical environments of carbon. The two major peaks at binding energies of 284.8 and $288.2 \mathrm{eV}$ correspond to the surface adventitious carbon and $\mathrm{sp}^{2}$-bonded carbon in $\mathrm{N}-\mathrm{C}=\mathrm{N}$ coordination [34]. Another weak peak at $286.2 \mathrm{eV}$ is identified as $\mathrm{sp}^{3}$-coordinated carbon bonds from defects on $g-\mathrm{C}_{3} \mathrm{~N}_{4}$
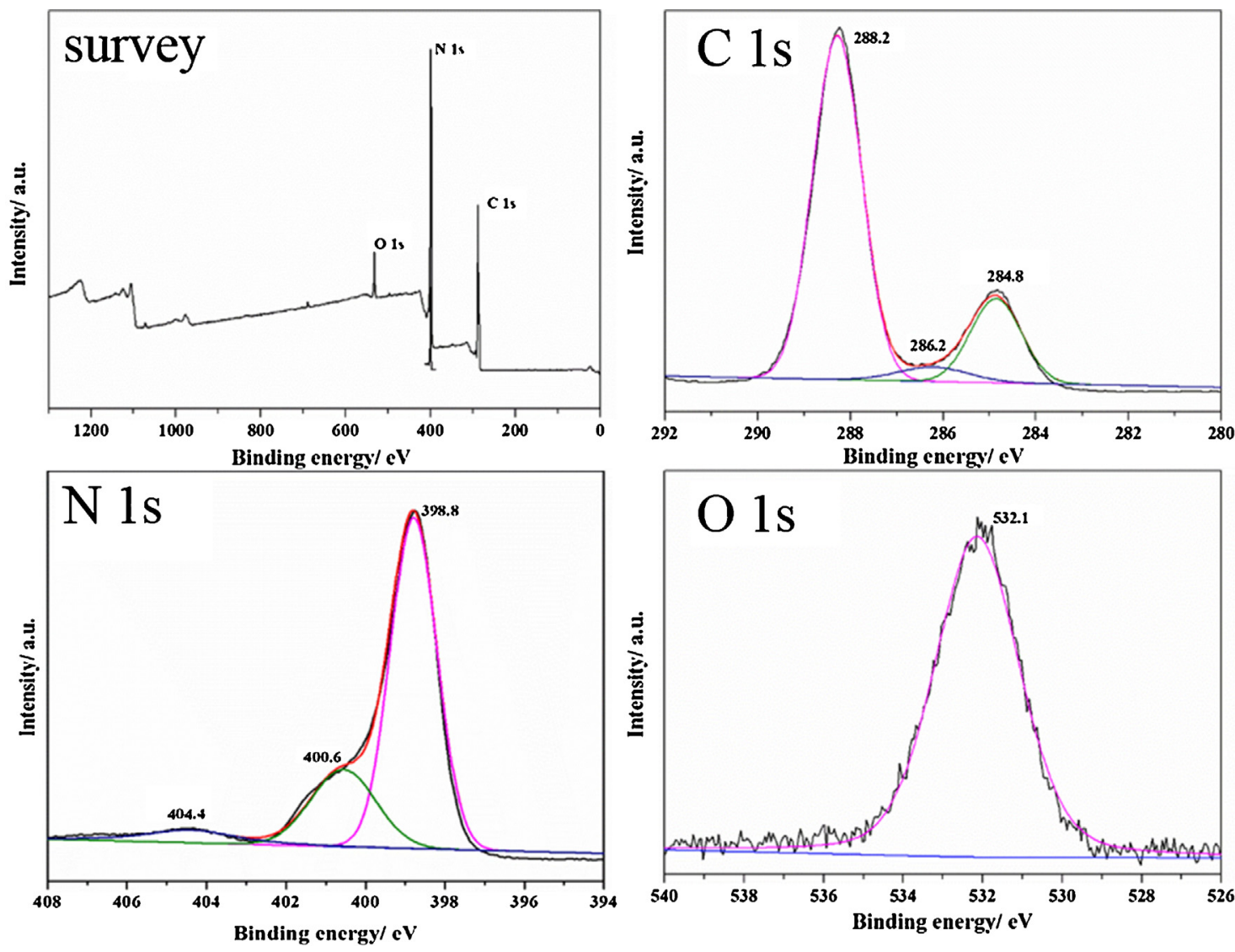

Fig. 2. XPS spectra of sample M1:1: (A) survey spectrum, high-resolution XPS spectrum of C1s, N1s, and O1s. 


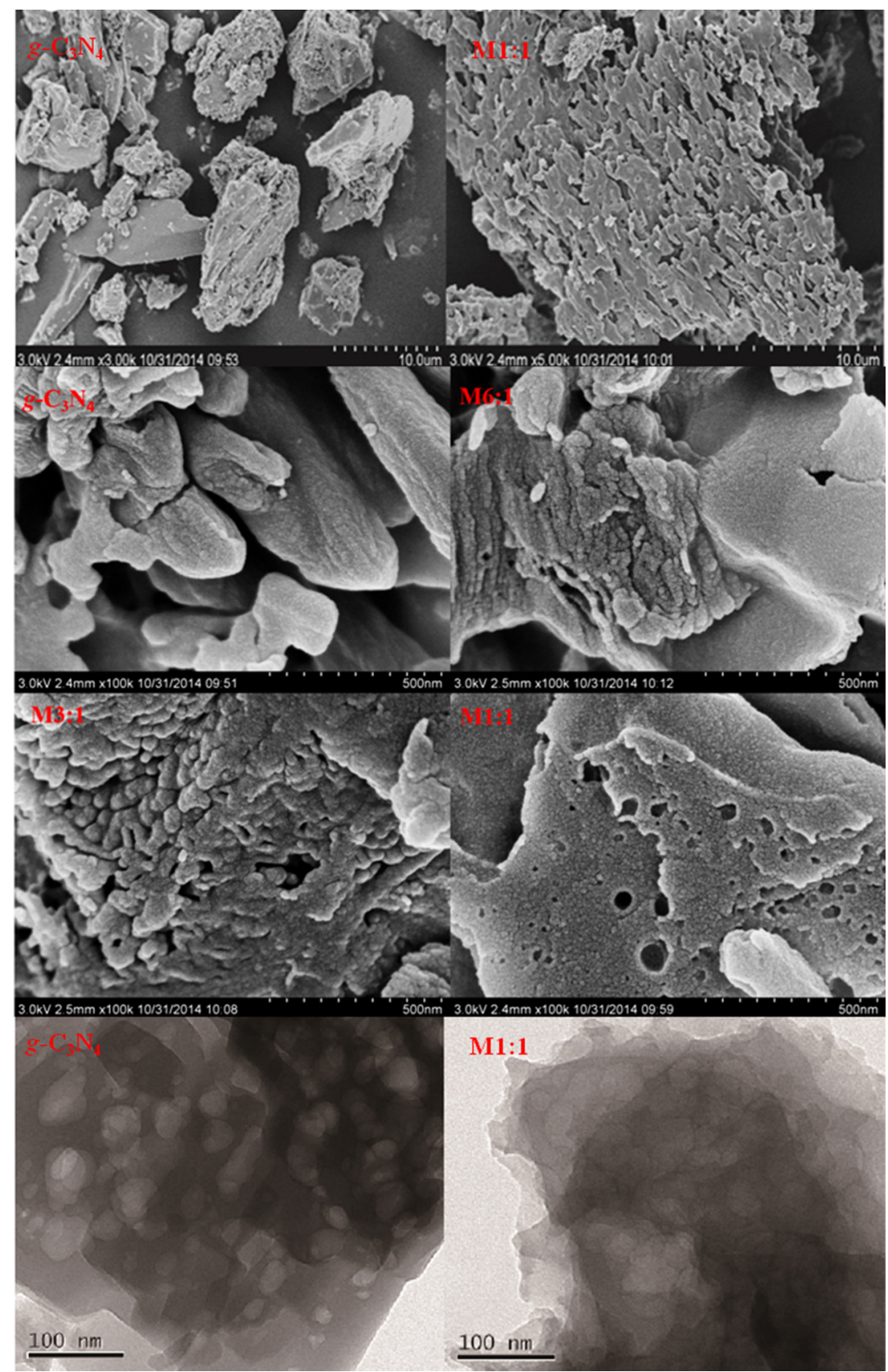

Fig. 3. SEM images of sample $g-C_{3} N_{4}$ and $M 1: 1$; enlarged SEM images of sample $g-C_{3} N_{4}, M 6: 1$, M3:1, and M1:1; TEM images of sample $g$ - $C_{3} N_{4}$ and $M 1: 1$.

surface [35]. The $\mathrm{N} 1 \mathrm{~s}$ spectrum shows three deconvoluted peaks at binding energies of 398.8, 400.6, and $404.4 \mathrm{eV}$. The first two signals are assigned to the nitrogen atoms in $\mathrm{N}-\mathrm{C}=\mathrm{N}$ and $\mathrm{N}-(\mathrm{C})_{3}$ moieties [24,35], and the last weak peak is attributed to charging effects $[34,36]$. The 01 s peak at 532.1 is relevant to the adsorbed $\mathrm{H}_{2} \mathrm{O}$ on surface [6].

The morphology and microstructure of obtained products by SEM and TEM images are shown in Fig. 3. As can be seen, the sample g- $\mathrm{C}_{3} \mathrm{~N}_{4}$ prepared without the addition of $\mathrm{HNO}_{3}$ displays disorderly stacked large and irregular clusters formed as the result of the arbitrary aggregation of small particles. The enlarged SEM image of sample $g-\mathrm{C}_{3} \mathrm{~N}_{4}$ shows that these large clusters have smooth surface without pores. The micropores and mesopores between nanosheets in sample $g-\mathrm{C}_{3} \mathrm{~N}_{4}$ (as shown in Fig. 4) are possibly created by the evolution of massive gases and splitting of the large layers during calcinations. The micropores were constructed by the aggregation of large clusters [33]. With the increase of $\mathrm{HNO}_{3}$ addition, more oxidative gases generated to oxidatively destruct bulk clusters into small particles and further into segments and nanosheets, thus improving the porosity of samples. Therefore, the size of particles was gradually reduced and the surface became coarse, depicted in the enlarged SEM images from $g-C_{3} \mathrm{~N}_{4}$ to M1:1. In addition, some small particles in sample M1:1 are regularly aggregated to produce tube-like units that are further assembled into large clusters along the direction perpendicular to the axis, as seen in SEM image of M1:1. It is significant that sample M1:1 is full of numerous mesopores from the channels of tube-like units on surface, revealing the possible enlarged specific surface area. It was reported that the white precipitate, the protonated melamine, showed tubular structures in ethylene glycol, owning to the electrostatic attraction, $\pi-\pi$ stacking interactions, and hydrogen bonding [25]. Upon pyrolysis, the tubular protonated melamine easily 


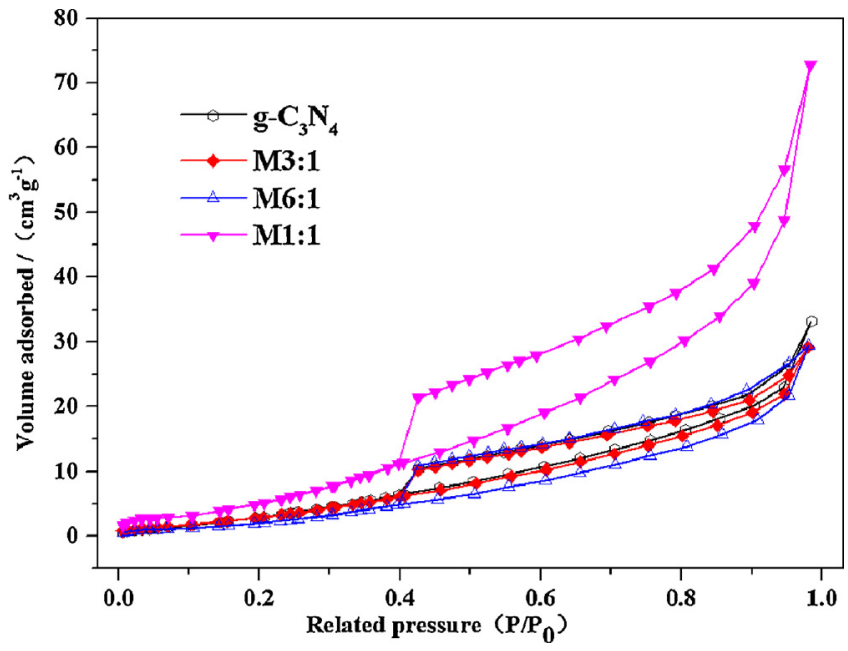

Fig. 4. Nitrogen adsorption-desorption isotherms of as-prepared $g-C_{3} N_{4}$ samples.

converted to tubular $g-C_{3} N_{4}$. During preparation, tubular geometry of protonated melamine could be stabilized and maintained in ethylene glycol, and partially destroyed in water [25]. As a result, partially destroyed tubular protonated melamine was further oxidized and gradually destructed to generate $\pi$-conjugated matrix g- $\mathrm{C}_{3} \mathrm{~N}_{4}$ with numerous pores on surface. Moreover, a lot of small pores generated in sample M6:1, M3;1, M1:1, and M1:3 (Fig. S1), instead of $g-\mathrm{C}_{3} \mathrm{~N}_{4}$, might be from the evolution of molecular oxygen and nitrogen oxides in the presence of sufficient amount of $\mathrm{HNO}_{3}$ at high temperature, since these gases likely oxidized and destroyed the microstructure surrounded. Besides, some arbitrary aggregated fragments were also identified by the quite transparent feature of the nanostructures in TEM image of sample M1: 1 .

Supplementary material related to this article can be found, in the online version, at http://dx.doi.org/10.1016/j.apsusc.2015.08. 124.

The elemental analysis data and specific surface areas of sample $g-C_{3} N_{4}$, M6:1, M3:1, M1:1, and M1:3 are listed in Table 1 . The atomic ratio of $C / N$ in sample $g-C_{3} N_{4}$ is 0.552 and much lower than the theoretical value 0.75 , attributing to the incomplete condensation of amine groups and the presence of structural defects [24]. With the increase of $\mathrm{HNO}_{3}$ addition, the atomic ratio of $\mathrm{C} / \mathrm{N}$ in samples is slightly improved and then reaches to a maximum 0.562 when the molar ratio of melamine $/ \mathrm{HNO}_{3}$ is set as 1 . This result can be mainly relevant to the large $\mathrm{N}$ loss as the result of the generation of large amount of oxidative gases as soon as the $\mathrm{HNO}_{3}$ addition is enhanced. However, when the molar ratio of melamine $/ \mathrm{HNO}_{3}$ is beyond $1: 1$, the atomic ratio $C / N$ is slight reduced and close to that of $g-C_{3} N_{4}$. A similar trend is also found in the variation of specific surface areas as below. As described in synthetic section, a white block was quickly formed. It is reasonable to speculate that some unreacted melamine were coprecipitated and might be enclosed by protonated melamine. Thereby, the produced sample M1:3 was $g-C_{3} N_{4}$ with a layer of modified $g-C_{3} N_{4}$, leading to a similar atomic ratio $C / N$ and specific surface area to those of $g-C_{3} N_{4}$. The nitrogen adsorption-desorption isotherms of as-prepared $g$ $\mathrm{C}_{3} \mathrm{~N}_{4}$ samples are displayed in Fig. 4 and can be classified as type

Table 1

Elemental analyses and specific surface areas of as-obtained samples.

\begin{tabular}{llllc}
\hline Samples & $\mathrm{C}($ at\%) & $\mathrm{N}($ at\%) & $C / N$ & $S_{\text {BET }}\left(\mathrm{m}^{2} / \mathrm{g}\right)$ \\
\hline$g-\mathrm{C}_{3} \mathrm{~N}_{4}$ & 33.274 & 60.234 & 0.552 & 5.3 \\
M6:1 & 32.198 & 58.830 & 0.547 & 4.1 \\
M3:1 & 33.119 & 59.479 & 0.557 & 6.4 \\
M1:1 & 32.187 & 57.283 & 0.562 & 11.8 \\
M1:3 & 33.051 & 59.658 & 0.554 & 4.7 \\
\hline
\end{tabular}

IV at the $P / P_{0}$ range of $0.4-1$ with hysteresis loops of type $\mathrm{H} 3$ [37], revealing the presence of mesopores and slit-like pores with diameters about $2-50 \mathrm{~nm}$ and over $50 \mathrm{~nm}$, respectively. These slit-like pores are associated to the aggregates of primary particles, which is accordance with SEM images. In addition, the adsorption ability of sample M1:1 is the strongest among those of all samples under identical conditions. Correspondingly, the specific surface area of sample M1:1 $11.8 \mathrm{~m}^{2} / \mathrm{g}$ is the largest one that is nearly twice as much as that of sample $g-\mathrm{C}_{3} \mathrm{~N}_{4}$, possibly due to the greatly improved porosity of sample M1:1.

The optical property of obtained samples was measured by UV-vis DRS analyses, as shown in Fig. 5. It is clear that $g-C_{3} N_{4}$ samples are photoresponsive in both UV and visible light regions, with adsorption edges at about $450 \mathrm{~nm}-465 \mathrm{~nm}$. The band gap energy $\left(E_{\mathrm{g}}\right)$ of these samples could be calculated by an empirical equation $\alpha h v=A\left(h v-E_{\mathrm{g}}\right)^{n / 2}$, where $\alpha, h v$, and $A$ are absorption coefficient, photon energy, and a constant, respectively [38]. $n$ was adopted as 4 because of the direct transition of $g-\mathrm{C}_{3} \mathrm{~N}_{4}$ samples [14,39], by which $E_{\mathrm{g}}$ values were estimated ranging from 2.60 to $2.76 \mathrm{eV}$. Adsorption edge of sample M1:1 is slightly blue shifted in comparison with that of sample $g-\mathrm{C}_{3} \mathrm{~N}_{4}$, corresponding to the slight expansion of band gap from $g-C_{3} N_{4} 2.64$ to $\mathrm{M} 1: 1 \mathrm{M} 2.67 \mathrm{eV}$, which is possibly attributed to the quantum size effect from the fragments generated in the oxidative environment [8] and some other effects, such as variable morphology, crystal size, and specific structure.

Photocatalytic evaluation of as-prepared $g-\mathrm{C}_{3} \mathrm{~N}_{4}$ samples was conducted by means of dye RhB degradation under visible-light irradiation $(\lambda>420 \mathrm{~nm})$. As shown in Fig. 6a, the decrease of initial concentration of RhB by direct photolysis is negligible, indicating the sufficient stability of $\mathrm{RhB}$ at tested conditions. The sample $g-\mathrm{C}_{3} \mathrm{~N}_{4}$ formed without $\mathrm{HNO}_{3}$ during synthesis shows an unsatisfactory removal efficiency of $40 \%$ that is similar to that of M1:3 and far lower than those of samples M1:1 and M3:1. It is obvious that the introduction of $\mathrm{HNO}_{3}$ in synthetic procedure is beneficial to construct samples with significantly improved photocatalytic capabilities. Especially, the addition amount of $\mathrm{HNO}_{3}$ is increased and catalytic ability of samples is accordingly enhanced. The best candidate, sample M1:1, could completely remove RhB in aqueous solution after $100 \mathrm{~min}$ under visible light irradiation and correspondingly, the solution was decolorized. The experimental data were subsequently fitted by a pseudo-first-order model to study reaction kinetics of the $\mathrm{RhB}$ degradation and the corresponding apparent reaction constants $(k)$ are shown in Fig. $6 \mathrm{~b}$. It is obvious that sample $\mathrm{M} 1: 1$ owns the best catalytic degradation ability, with a $k$ value 6.2 times that of unmodified $g-\mathrm{C}_{3} \mathrm{~N}_{4}$ sample. However, the introduction of excess amount of $\mathrm{HNO}_{3}$ would induce to generate samples, like M1:3, with relatively low catalytic capability. It is general that photocatalytic capability of materials is closely relevant to microstructures and band gap energies [40,41]. As discussed above, porous $g-\mathrm{C}_{3} \mathrm{~N}_{4}$ samples with enlarged specific surface areas could be fabricated in presence of a proper concentration of $\mathrm{HNO}_{3}$ solution. Below and over that concentration, the porosity and specific surface area of samples shrank. An enlarged specific surface area is able to increase the interfacial surface between catalysts and pollutant molecules and provide abundant active sites to contact with reactant molecules and hereby enhanced the visible-light harvesting [21]. In addition, the sufficient porosity of samples facilitates both the immigration of reactants and export of degraded products, and multiple scattering of visible light. The variation of band gaps of samples after pretreatment of $\mathrm{HNO}_{3}$ solution is slight, ensuring those samples with appropriate electronic structures. With the improved porosity, the largest specific surface area, and the suitable band gap, the sample M1:1 exhibits the best photocatalytic behavior among all tested samples.

From the VB XPS analysis in Fig. 7a, the valence band maximum $\left(E_{V B}\right)$ of sample $\mathrm{M} 1: 1$ is estimated at $1.87 \mathrm{eV}$ and correspondingly, 

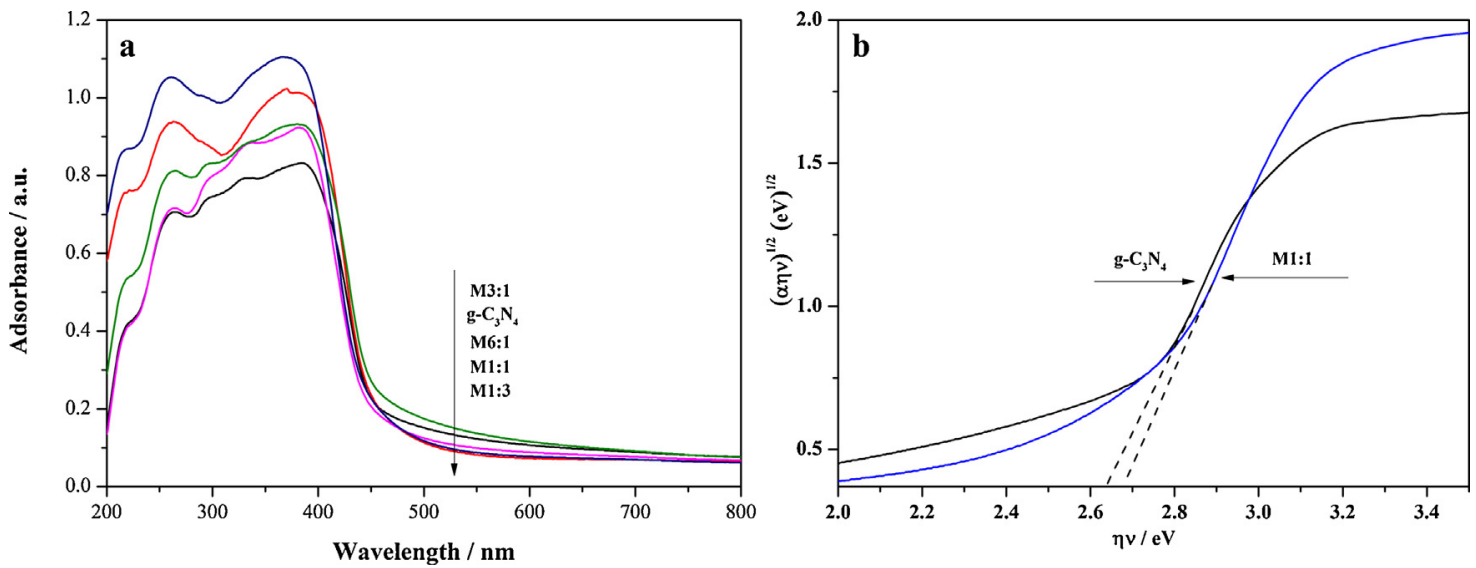

Fig. 5. UV-vis DRS spectra of $g-C_{3} \mathrm{~N}_{4}$ samples (a) and $(\alpha h v)^{1 / 2}$ versus $h v$ plots of sample $g-C_{3} \mathrm{~N}_{4}$ and $\mathrm{M} 1: 1$.
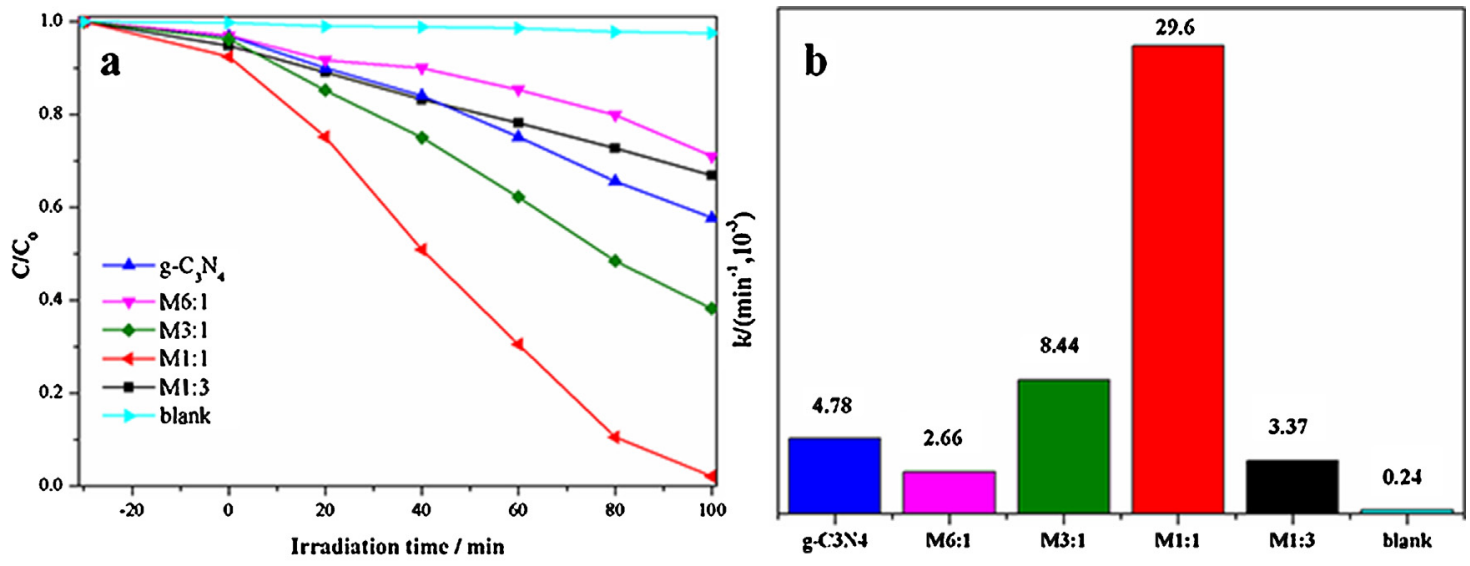

Fig. 6. Photocatalytic degradation of RhB by various $g-C_{3} N_{4}$ samples under visible light (a) and corresponding apparent reaction rates (b).

the conduction band minimum $\left(E_{C B}\right)$ is calculated as $-0.8 \mathrm{eV}$. A plausible photocatalytic degradation mechanism was proposed and displayed in Fig. 7b. Upon visible light irradiation $(420 \mathrm{~nm}<\lambda<780 \mathrm{~nm}$ ), sample M1:1 is able to be excited to generate electrons and holes that stay at the bottom of conduction band and top of valance band, respectively. Since the $E_{C B}(-0.8 \mathrm{eV})$ of sample $\mathrm{M} 1: 1$ is more negative than $E\left(\mathrm{O}_{2} /{ }^{\circ} \mathrm{O}_{2}{ }^{-}\right)(-0.33 \mathrm{eV})$ [42], the electrons tend to react with adsorbed oxygen to produce $\mathrm{O}_{2}{ }^{-}$radicals through an one-electron reaction. However, the $E_{V B}(+1.87 \mathrm{eV})$ is less positive than $E\left({ }^{\bullet} \mathrm{OH} / \mathrm{OH}^{-}\right)(+1.99 \mathrm{eV})$ and $E\left(\bullet \mathrm{OH} / \mathrm{H}_{2} \mathrm{O}\right)(+2.37 \mathrm{eV})[33,43]$ and holes are insufficient to directly oxidize surface water to form ${ }^{\bullet} \mathrm{OH}$ radicals. ${ }^{\bullet} \mathrm{OH}$ radicals can be formed through a two-electron oxygen reduction route instead [44]. The possibility of generating ${ }^{-} \mathrm{O}_{2}{ }^{-}$and $\bullet \mathrm{OH}$ radicals were also measured by ESR with DMPO spin-trap analysis in Fig. 8. Signals of DMPO- $\bullet \mathrm{OH}$ adduct can be weakly detectable, and six characteristic peaks of DMPO- $\bullet^{-} \mathrm{O}_{2}^{-}$adduct are easy to be observed, indicative of the formation of both $\bullet^{-\mathrm{O}_{2}}$ and $\bullet \mathrm{OH}^{-}$radicals during catalytic reactions [45]. As a result, the formed $\bullet^{\circ} \mathrm{O}_{2}{ }^{-}$radicals together with photogenerated holes are deemed as dominant active radicals and $\bullet \mathrm{OH}$ radicals play minor roles for the degradation of RhB molecules.
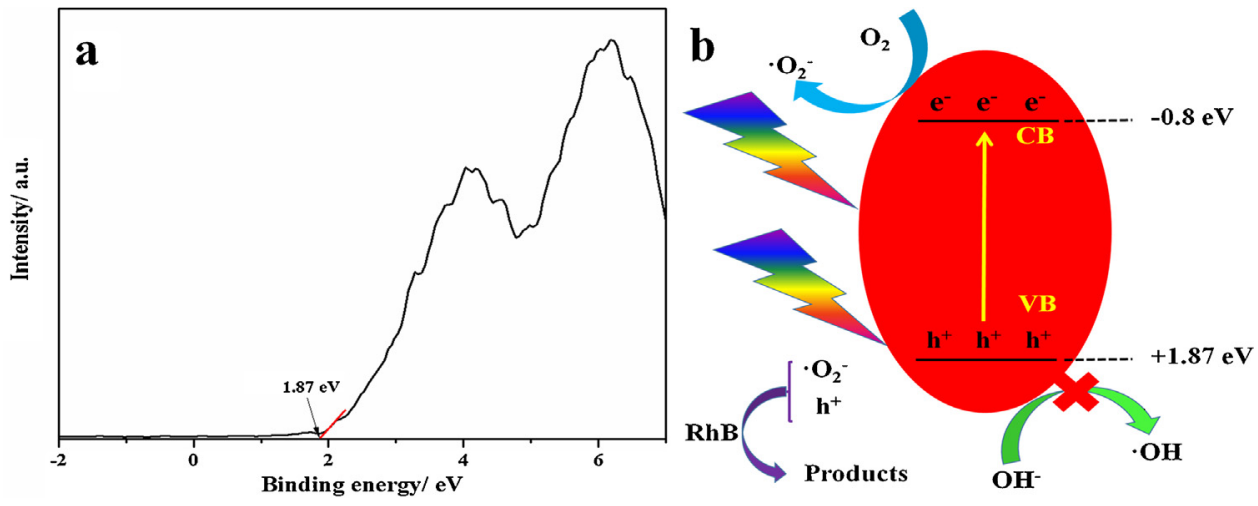

Fig. 7. VB XPS analysis of sample M1:1 (a) and proposed mechanism upon visible-light irradiation (b). 

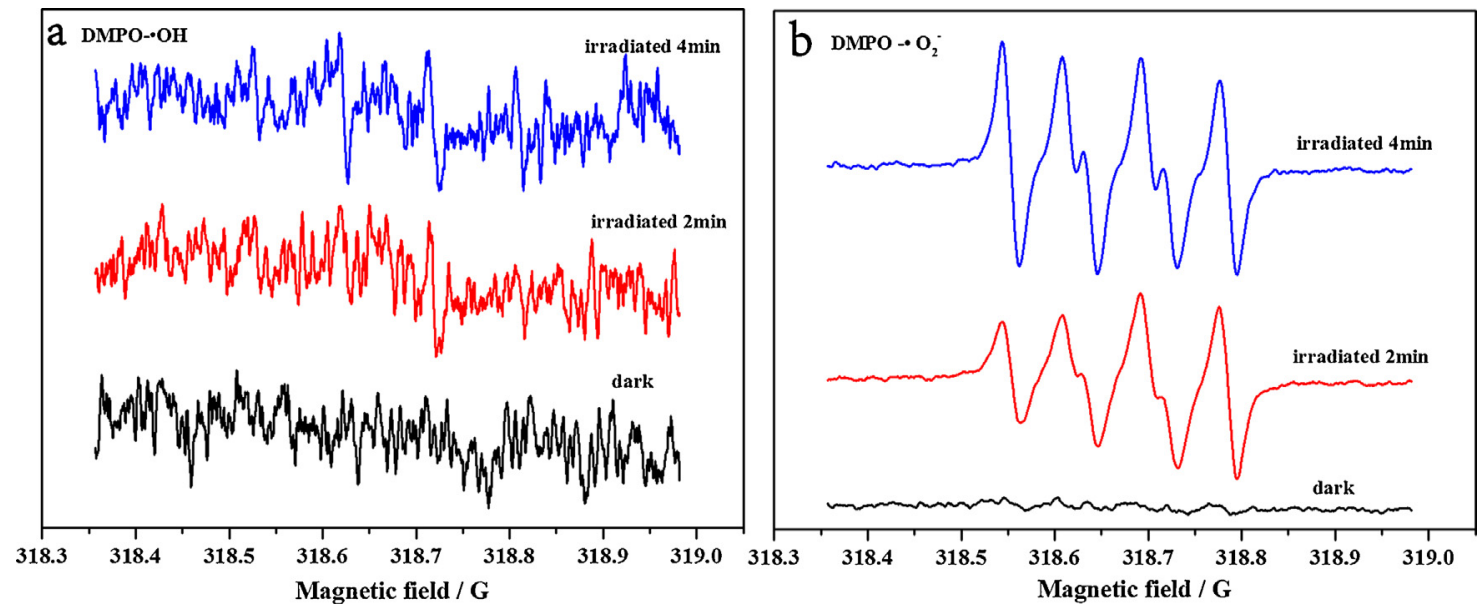

Fig. 8. DMPO spin-trapping ESR spectra of sample M1:1 for DMPO-・OH (a) and DMPO- $\mathrm{O}_{2}{ }^{-}$(b).

\section{Conclusion}

In this study, a series of porous $g-\mathrm{C}_{3} \mathrm{~N}_{4}$ samples were constructed via a facile direct pyrolysis of $\mathrm{HNO}_{3}$-treated melamine and subsequently characterized by various analytical techniques. Obviously, an appropriate amount of nitric acid involved facilitated to generate samples with the improved porosity and enlarged specific surface areas. These modified porous samples displayed quite higher catalytic removal efficiency than unmodified $g-C_{3} N_{4}$ under visible light irradiation. In particularly, the best candidate M1:1 could completely decompose RhB after $100 \mathrm{~min}$, with an apparent reaction rate approximately 6.2 times that of $g-C_{3} N_{4}$. The enhancement of photocatalytic performance mainly attributed to the porous structure with the enlarged specific surface area and the suitable band gap energy. Finally, a possible photocatalytic mechanism was proposed basing upon ESR measurements.

\section{Acknowledgements}

We are grateful to the National Natural Science Foundation of China (Grant number 21207089 and 41230858), the projectsponsored by SRF for ROCS, SEM, and the Hujiang Foundation of China (B14003) for financial support.

\section{References}

[1] S.W. Cao, J.X. Low, J.G. Yu, M. Jaroniec, Polymeric photocatalysts based on graphitic carbon nitride, Adv. Mater. 27 (2015) 2150-2176.

[2] G. Dong, Y. Zhang, Q. Pan, J. Qiu, A fantastic graphitic carbon nitride $\left(g-\mathrm{C}_{3} \mathrm{~N}_{4}\right)$ material: electronic structure, photocatalytic and photoelectronic properties, J. Photochem. Photobiol. C 20 (2014) 33-50.

[3] X. Wang, K. Maeda, A. Thomas, K. Takanabe, G. Xin, J.M. Carlsson, K. Domen, M. Antonietti, A metal-free polymeric photocatalyst for hydrogen production from water under visible light, Nat. Mater. 8 (2009) 76-80.

[4] Z. Zhao, Y. Sun, F. Dong, Graphitic carbon nitride based nanocomposites: a review, Nanoscale 7 (2015) 15-37.

[5] J.S. Zhang, B. Wang, X.C. Wang, Carbon nitride polymeric semiconductor for photocatalysis, Prog. Chem. 26 (2014) 19-29.

[6] S.W. Cao, Y.P. Yuan, J. Barber, S.C.J. Loo, C. Xue, Noble-metal-free $g-\mathrm{C}_{3} \mathrm{~N}_{4} / \mathrm{Ni}(\mathrm{dmgH})_{2}$ composite for efficient photocatalytic hydrogen evolution under visible light irradiation, Appl. Surf. Sci. 319 (2014) 344-349.

[7] B. Zhu, P. Xia, W. Ho, J. Yu, Isoelectric point and adsorption activity of porous g- $\mathrm{C}_{3} \mathrm{~N}_{4}$, Appl. Surf. Sci. 344 (2015) 188-195

[8] F. Dong, Y. Li, Z. Wang, W.K. Ho, Enhanced visible light photocatalytic activity and oxidation ability of porous graphene-like $g-C_{3} N_{4}$ nanosheets via thermal exfoliation, Appl. Surf. Sci. (2015), http://dx.doi.org/10.1016/j.apsusc.04.034.

[9] M. Zhang, X. Bai, D. Liu, J. Wang, Y. Zhu, Enhanced catalytic activity of potassium-doped graphitic carbon nitride induced by lower valence position, Appl. Catal. B 164 (2015) 77-81.

[10] Y. Zhou, L. Zhang, J. Liu, X. Fan, B. Wang, M. Wang, W. Ren, J. Wang, M. Li, J. Shi, Brand new P-doped $g$ - $\mathrm{C}_{3} \mathrm{~N}_{4}$ : enhanced photocatalytic activity for $\mathrm{H}_{2}$ evolution and Rhodamine B degradation under visible light, J. Mater. Chem. A 3 (2015) 3862-3867.

[11] J. Hong, X. Xia, Y. Wang, R. Xu, Mesoporous carbon nitride with in situ sulfur doping for enhanced photocatalytic hydrogen evolution from water under visible light, J. Mater. Chem. 22 (2012) 15006-15012.

[12] K. Wang, Q. Li, B. Liu, B. Cheng, W. Ho, J. Yu, Sulfur-doped $g-C_{3} \mathrm{~N}_{4}$ with enhanced photocatalytic $\mathrm{CO}_{2}$-reduction performance, Appl. Catal. B 176 (2015) 44-52.

[13] S. Hu, L. Ma, J. You, F. Li, Z. Fan, G. Lu, D. Liu, J. Gui, Enhanced visible light photocatalytic performance of $g-\mathrm{C}_{3} \mathrm{~N}_{4}$ photocatalysts co-doped with iron and phosphorus, Appl. Surf. Sci. 311 (2014) 164-171.

[14] F. Dong, Z. Wang, Y. Sun, W.-K. Ho, H. Zhang, Engineering the nanoarchitecture and texture of polymeric carbon nitride semiconductor for enhanced visible light photocatalytic activity, J. Colloid Interface Sci. 401 (2013) 70-79.

[15] Q. Gu, Y. Liao, L. Yin, J. Long, X. Wang, C. Xue, Template-free synthesis of porous graphitic carbon nitride microspheres for enhanced photocatalytic hydrogen generation with high stability, Appl. Catal. B 165 (2015) 503-510.

[16] B. Shen, Z. Hong, Y. Chen, B. Lin, B. Gao, Template-free synthesis of a novel porous $g$ - $\mathrm{C}_{3} \mathrm{~N}_{4}$ with 3D hierarchical structure for enhanced photocatalytic $\mathrm{H}_{2}$ evolution, Mater. Lett. 118 (2014) 208-211.

[17] K. Dai, L. Lu, C. Liang, Q. Liu, G. Zhu, Heterojunction of facet coupled $g-\mathrm{C}_{3} \mathrm{~N}_{4}$ /surface-fluorinated $\mathrm{TiO}_{2}$ nanosheets for organic pollutants degradation under visible LED light irradiation, Appl. Catal. B 156 (2014) $331-340$.

[18] S. Kumar, T. Surendar, A. Baruah, V. Shanker, Synthesis of a novel and stable g- $\mathrm{C}_{3} \mathrm{~N}_{4}-\mathrm{Ag}_{3} \mathrm{PO}_{4}$ hybrid nanocomposite photocatalyst and study of the photocatalytic activity under visible light irradiation, J. Mater. Chem. A 1 (2013) 5333-5340.

[19] G. Liao, S. Chen, X. Quan, H. Yu, H. Zhao, Graphene oxide modified $g-C_{3} N_{4}$ hybrid with enhanced photocatalytic capability under visible light irradiation, J. Mater. Chem. 22 (2012) 2721-2726.

[20] C.Z. Zheng, C.Y. Zhang, G.H. Zhang, D.J. Zhao, Y.Z. Wang, Enhanced photocatalytic performance of $g-\mathrm{C}_{3} \mathrm{~N}_{4}$ with $\mathrm{BiOCl}$ quantum dots modification, Mater. Res. Bull. 55 (2014) 212-215.

[21] F. Chang, J. Zhang, Y. Xie, J. Chen, C. Li, J. Wang, J. Luo, B. Deng, X. Hu, Fabrication, characterization, and photocatalytic performance of exfoliated $g-\mathrm{C}_{3} \mathrm{~N}_{4}-\mathrm{TiO}_{2}$ hybrids, Appl. Surf. Sci. 311 (2014) 574-581.

[22] H. Yu, F. Chen, F. Chen, X. Wang, In situ self-transformation synthesis of $g-C_{3} \mathrm{~N}_{4}$-modified CdS heterostructure with enhanced photocatalytic activity, Appl. Surf. Sci. (2015), http://dx.doi.org/10.1016/j.apsusc.2015.06.074.

[23] G. Dong, L. Zhang, Porous structure dependent photoreactivity of graphitic carbon nitride under visible light, J. Mater. Chem. 22 (2012) 1160-1166.

[24] H. Yan, Y. Chen, S. Xu, Synthesis of graphitic carbon nitride by directly heating sulfuric acid treated melamine for enhanced photocatalytic $\mathrm{H}_{2}$ production from water under visible light, Int. J. Hydrogen Energy 37 (2012) 125-133.

[25] J. Gao, Y. Zhou, Z. Li, S. Yan, N. Wang, Z. Zou, High-yield synthesis of millimetre-long, semiconducting carbon nitride nanotubes with intense photoluminescence emission and reproducible photoconductivity, Nanoscale 4 (2012) 3687-3692.

[26] M. Tahir, C. Cao, F.K. Butt, F. Idrees, N. Mahmood, Z. Ali, I. Aslam, M. Tanveer, M. Rizwan, T. Mahmood, Tubular graphitic- $\mathrm{C}_{3} \mathrm{~N}_{4}$ : a prospective material for energy storage and green photocatalysis, J. Mater. Chem. A 1 (2013) 13949-13955

[27] M. Tahir, C. Cao, N. Mahmood, F.K. Butt, A. Mahmood, F. Idrees, S. Hussain, M. Tanveer, Z. Ali, I. Aslam, Multifunctional $g-C_{3} N_{4}$ nanofibers: a template-free fabrication and enhanced optical, electrochemical, and photocatalyst properties, ACS Appl. Mater. Interfaces 6 (2014) 1258-1265.

[28] Y. Zhong, Z. Wang, J. Feng, S. Yan, H. Zhang, Z. Li, Z. Zou, Improvement in photocatalytic $\mathrm{H}_{2}$ evolution over $\mathrm{g}-\mathrm{C}_{3} \mathrm{~N}_{4}$ prepared from protonated melamine, Appl. Surf. Sci. 295 (2014) 253-259. 
[29] B. Amin, H.M. Feriz, A.T. Hariri, N.T. Meybodi, H. Hosseinzadeh, Protective effects of the aqueous extract of crocus sativus against ethylene glycol induced nephrolithiasis in rats, EXCLI J. 14 (2015) 411-422.

[30] F. Chang, Y. Xie, C. Li, J. Chen, J. Luo, X. Hu, J. Shen, A facile modification of $g-\mathrm{C}_{3} \mathrm{~N}_{4}$ with enhanced photocatalytic activity for degradation of methylene blue, Appl. Surf. Sci. 280 (2013) 967-974.

[31] X.H. Li, J. Zhang, X. Chen, A. Fischer, A. Thomas, M. Antonietti, X. Wang, Condensed graphitic carbon nitride nanorods by nanoconfinement: promotion of crystallinity on photocatalytic conversion, Chem. Mater. 23 (2011) 4344-4348.

[32] S.C. Yan, Z.S. Li, Z.G. Zou, Photodegradation performance of $g-\mathrm{C}_{3} \mathrm{~N}_{4}$ fabricated by directly heating melamine, Langmuir 25 (2009) 10397-10401.

[33] F. Dong, Z. Wang, Y. Li, W.-K. Ho, S.C. Lee, Immobilization of polymeric $g-C_{3} N_{4}$ on structured ceramic foam for efficient visible light photocatalytic air purification with real indoor illumination, Environ. Sci. Technol. 48 (2014) 10345-10353.

[34] J. Liu, T. Zhang, Z. Wang, G. Dawson, W. Chen, Simple pyrolysis of urea into graphitic carbon nitride with recyclable adsorption and photocatalytic activity, J. Mater. Chem. 21 (2011) 14398-14401.

[35] S.W. Cao, Y.P. Yuan, J. Fang, M.M. Shahjamali, F.Y.C. Boey, J. Barber, S.C.J. Loo, C. Xue, In-situ growth of CdS quantum dots on $g-\mathrm{C}_{3} \mathrm{~N}_{4}$ nanosheets for highly efficient photocatalytic hydrogen generation under visible light irradiation, Int. J. Hydrogen Energy 38 (2013) 1258-1266.

[36] G. Li, N. Yang, W. Wang, W.F. Zhang, Synthesis, photophysical and photocatalytic properties of $\mathrm{N}$-doped sodium niobate sensitized by carbon nitride, J. Phys. Chem. C 113 (2009) 14829-14833.

[37] M. Kruk, M. Jaroniec, Gas adsorption characterization of ordered organic-inorganic nanocomposite materials, Chem. Mater. 13 (2001) 3169-3183.
[38] S.J. Hong S. Lee, J.S. Jang J.S. Lee, Heterojunction BiVO4/WO3 electrodes for enhanced photoactivity of water oxidation, Energy Environ. Sci 4 (2011) 1781-1787.

[39] S. Yang, Y. Gong, J. Zhang, L. Zhan, L. Ma, Z. Fang, R. Vajtai, X. Wang, P.M. Ajayan, Exfoliated graphitic carbon nitride nanosheets as efficient catalysts for hydrogen evolution under visible light, Adv. Mater. 25 (2013) 2452-2456.

[40] Y. Hong, S.L. Wang, L.Q. Li, B. Xu, G.Q. Han, Photocatalytic degradation performance of zinc mixing ZnO nano-rod, J. Univ. Shanghai Sci. Tech. 32 (2010) 159-162.

[41] H.Y. Xu, S.L. Zhou, H. Tao, X.H. Cai, J.S.D. Zeng, Review of research advancements on degradation of phthalate esters in sewage, J. Univ. Shanghai Sci. Tech. 32 (2010) 418-422.

[42] S. Wang, D. Li, C. Sun, S. Yang, Y. Guan, H. He, Synthesis and characterization of g- $\mathrm{C}_{3} \mathrm{~N}_{4}-\mathrm{Ag}_{3} \mathrm{PO}_{4}$ composites with significantly enhanced visible-light photocatalytic activity for triphenyl methane dye degradation, Appl. Catal. B 144 (2014) 885-892.

[43] N. Boonprakob, N. Wetchakun, S. Phanichphant, D. Waxler, P. Sherrell, A Nattestad, J. Chen, B. Inceesungvorn, Enhanced visible-light photocatalytic activity of $g-\mathrm{C}_{3} \mathrm{~N}_{4} / \mathrm{TiO}_{2}$ films, J. Colloid Interface Sci. 417 (2014) 402-409.

[44] Z. Wang, W. Guan, Y. Sun, F. Dong, Y. Zhou, W.K. Ho, Water-assisted production of honeycomb-like $g-C_{3} \mathrm{~N}_{4}$ with ultralong carrier lifetime and outstanding photocatalytic activity, Nanoscale 7 (2015) 2471-2479.

[45] F.T. Li, Q. Wang, X.J. Wang, B. Li, Y.J. Hao, R.H. Liu, D.S. Zhao, In-situ one-step synthesis of novel $\mathrm{BiOCl} / \mathrm{Bi}_{24} \mathrm{O}_{31} \mathrm{Cl}_{10}$ heterojunctions via self-combustion of ionic liquid with enhanced visible-light photocatalytic activities, Appl. Catal. B 150 (2014) 574-584 